\title{
Crowdsensing Task Assignment Based on Particle Swarm Optimization in Cognitive Radio Networks
}

\author{
Linbo Zhai ${ }^{1,2}$ and Hua Wang ${ }^{2}$ \\ ${ }^{1}$ Shandong Provincial Key Laboratory for Distributed Computer Software Novel Technology, Shandong Normal University, \\ Jinan, China \\ ${ }^{2}$ School of Computer Science and Technology, Shandong University, Jinan, China \\ Correspondence should be addressed to Linbo Zhai; zhai@mail.sdu.edu.cn
}

Received 26 April 2017; Revised 18 July 2017; Accepted 2 August 2017; Published 11 September 2017

Academic Editor: Bin Guo

Copyright (C) 2017 Linbo Zhai and Hua Wang. This is an open access article distributed under the Creative Commons Attribution License, which permits unrestricted use, distribution, and reproduction in any medium, provided the original work is properly cited.

Cognitive radio technology allows unlicensed users to utilize licensed wireless spectrum if the wireless spectrum is unused by licensed users. Therefore, spectrum sensing should be carried out before unlicensed users access the wireless spectrum. Since mobile terminals such as smartphones are more and more intelligent, they can sense the wireless spectrum. The method that spectrum sensing task is assigned to mobile intelligent terminals is called crowdsourcing. For a large-scale region, we propose the crowdsourcing paradigm to assign mobile users the spectrum sensing task. The sensing task assignment is influenced by some factors including remaining energy, locations, and costs of mobile terminals. Considering these constraints, we design a precise sensing effect function with a local constraint and aim to maximize this sensing effect to address crowdsensing task assignment. The problem of crowdsensing task assignment is difficult to solve since we prove that it is NP-hard. We design an optimal algorithm based on particle swarm optimization to solve this problem. Simulation results show our algorithm achieves higher performance than the other algorithms.

\section{Introduction}

In recent years, the wireless traffic has grown heavily and this case leads to crowd wireless spectrum. According to the current policy that wireless spectrum assignment is fixed, only licensed users can utilize the licensed wireless spectrum. Even though the wireless spectrum is idle, unlicensed users cannot use the idle spectrum. Therefore, the current policy of spectrum assignment leads to low ratio of wireless spectrum utilization. To solve this problem, cognitive radio has recently emerged to improve wireless spectrum utilization [1]. When the licensed wireless spectrum is idle, cognitive radio makes unlicensed users utilize the wireless spectrum. Therefore, unlicensed users should carry out spectrum sensing before they use the wireless spectrum.

With the development of mobile terminals such as smartphones and pads, a new paradigm called mobile crowd sensing and computing (MCSC) appears [2]. The formal definition of MCSC is described as follows: a new sensing paradigm that empowers ordinary citizens to contribute data sensed or generated from their mobile devices and aggregates and fuses the data in the cloud for crowd intelligence extraction and human-centric service delivery.

Inspired by MCSC, mobile terminals configured with sensors are leveraged to accomplish spectrum sensing task. In the same spirit, with the recent Federal Communications Commission (FCC) ruling that a geolocation database could be used by Secondary TV spectrum users to obtain the spectrum availability, it is assumed that there is a crowdsourcingbased fusion center (FC). FC assigns sensing task to mobile users and receives the sensing data from them. To incentivize mobile users to carry out sensing tasks, FC needs to provide monetary benefits. This way is called crowdsourcing.

In this paper, we propose the crowdsourcing paradigm to assign the spectrum sensing task to many mobile users. It is assumed that there is a crowdsourcing-based fusion center (FC). FC assigns the sensing task to mobile users. During the assignment process, we have considered some factors. 
At first, the remaining energy is very important to mobile users. Only when a mobile user has enough energy can the wireless spectrum be sensed. Then mobile users should be given incentives to carry out spectrum sensing. With a limited budget, FC may choose a subset of whole mobile users to carry out spectrum sensing. At last, the positions of mobile users also influence the sensing results. Considering these factors, we propose precise sensing effect function for the crowdsourcing-based sensing task assignment. And the objective function considers a local constraint. Then we prove that the sensing task assignment is NP-hard. Therefore, we design an optimal algorithm based on particle swarm optimization (PSO) to solve the problem. Simulation results show our proposed algorithm achieves higher performance than other algorithms.

In this paper, we study the problem of sensing task assignment. The main contributions of this paper are summarized below.

(i) Considering the remaining energy of mobile users, budget constraint, and mobile users' positions, we propose precise objective function with a local constraint. We define the local constraint which means the sensing effect of a channel in a location is not less than a threshold. Compared to other literatures, we aim to not only maximize global sensing effect but also satisfy the local sensing constraint. And we prove the sensing task assignment is NP-hard.

(ii) Since the sensing task assignment is NP-hard, we design an optimal algorithm based on particle swarm optimization (PSO) to solve the problem. To the best of our knowledge, there is no related work designing the PSO-based algorithm to solve sensing task assignment in cognitive radio networks.

(iii) Simulation results show our proposed algorithm achieves higher performance than other algorithms.

The rest of the paper is organized as follows. In Section 2, related literatures are introduced. In Section 3, the system model of sensing task assignment is described. In Section 4, we design a PSO-based algorithm to solve the sensing task assignment. In Section 5, the proposed algorithm is evaluated with simulation results. Finally, conclusions are shown in Section 6.

\section{Related Work}

In cognitive radio networks, licensed users activity will decide whether the spectrum is idle or not [3]. As some factors such as shadowing and multipath fading may make a user mistake the sensing result, cooperative spectrum sensing is proposed to improve the sensing accuracy [4].

There have been some related literatures about cooperative spectrum sensing. In wideband wireless system, users exchange their compressed sensing results. According to the sensing results, they estimate the spectrum states cooperatively [5]. In [6], authors propose a two-level defense scheme to solve the attackers in cooperative spectrum sensing. In [7], cooperative spectrum sensing based on crowdsourcing is studied to address the security issue brought by malicious mobile users. In [8], authors consider the simultaneous sensing and transmitting of users and propose a novel detection model for cooperative spectrum sensing. In multichannel networks, the sensing task assignment is considered in parallel, and several sensing strategies are proposed to schedule users based on network parameters [9]. In [10], authors propose a game-theoretic distributed power control mechanism based on channel sensing results of users in cognitive wireless sensor network. To maximize the sensing quality, authors study the problem of multichannel sensing assignment in the multichannel system [11-13]. These literatures use a simplistic objective function and there is no budget constraint. If the system has a limited budget, there may be only a subset of mobile users chosen to carry out spectrum sensing. In [14], considering budget constraint, the authors study the problem of sensing task and channel allocation. However, the energy of mobile users is not considered. In [15], considering the character of sensing tasks and the sensor availability, authors study the multitask allocation problem to maximize overall system utility. It is the first to study different data quality metrics and formulate the multitask allocation optimization problem when diverse sensing capability constraints of each participant are taken into account. To achieve the nearoptimal objective, the method using a two-phase offline multitask allocation framework needs historical call data from the telecom operator.

The aforementioned literatures use centralized algorithms. There are some distributed methods about spectrum sensing. In [16], with a distributed way, spatial spectrum sensing is studied to make use of spatial spectrum opportunities. To analyze the performance of spatial spectrum sensing, stochastic geometry is utilized. In [10], based on channel sensing results of users, a game-theoretic distributed power control mechanism is proposed. Besides, there are other studies about spectrum sensing [17-20].

\section{System Model}

It is assumed that there is a crowdsourcing-based fusion center (FC). FC assigns the sensing task to mobile users. Remaining energy and positions of mobile users, as well as limited budget, may influence the assignment process. Considering these constraints, we propose precise sensing effect function with a local constraint. Then we prove the sensing task assignment is NP-hard.

3.1. Problem Formulation. We assume that there are many locations needed to be sensed. In each location, there are many channels that needed sensing. By crowdsensing task assignment, we aim to maximize the sensing effect with a local constraint.

Let $M$ denote the number of locations needed to be sensed and $N(j)$ denote the number of channels that should be sensed in a location $j$. In the location $j$, shadowing, multipath fading, and other issues may influence the sensing results of mobile users in different positions of this location. In other words, mobile users may obtain different sensing results in the same location since they are at different 
positions. Therefore, location $j$ may be divided into several sublocations. The spatial diversity can be captured by the sensing outcomes of mobile users in different sublocations. In a sublocation $h$ of location $j, z_{h j}^{i}=1$ denotes that there is at least one mobile user sensing channel $i$, and $z_{h j}^{i}=$ 0 denotes that there are no mobile users sensing channel $i$. In location $j$, let $y_{j}^{i}$ denote the number of sublocations where channel $i$ is sensed by at least one mobile user. We can derive $y_{j}^{i}=\sum_{h=1}^{m(j)} z_{h j}^{i}$, where $m(j)$ denotes the number of sublocations in location $j$. Obviously, the higher $y^{i}{ }_{j}$ is, the more effective the sensing result is. When $y_{j}^{i}$ equals zero, there is no sensing effect. When $y_{j}^{i}$ equals $m(j)$, the maximized sensing effect is reached. We can imagine that sensing effect increases fast as $y_{j}^{i}$ increases when $y^{i}{ }_{j}$ is small, while sensing effect increases slowly as $y^{i}{ }_{j}$ increases when $y^{i}{ }_{j}$ is large. Let $f(i, j)=\sqrt{y^{i}{ }_{j} / m(j)}$ denote the sensing effect of channel $i$ in location $j$. Then we can design the sensing effect function for the crowdsensing task assignment as follows:

$$
\sum_{j=1}^{M} \sum_{i=1}^{N(j)} w_{j}^{i} f(i, j)
$$

where $w^{i}{ }_{j}$ denotes the nonnegative weight with $\sum_{j=1}^{M} \sum_{i=1}^{N(j)} w_{j}^{i}=1$, and $w_{j}^{i}$ could distinguish the important degrees of sensing channels in each location. According to formula (1), the sensing effect function increases as $y^{i}{ }_{j}$ varies from zero to $m(j)$. And the smaller $y_{j}{ }_{j}$ is, the faster sensing effect function increases with the $y_{j}^{i}$ growth. The larger $y_{j}^{i}$ is, the more slowly sensing effect function increases with the $y_{j}^{i}$ growth.

To obtain optimized sensing effect, we aim to maximize the sensing effect function in (1) with a local constraint which means the sensing effect of channel $i$ in location $j$ is no less than a threshold $H$. The local constraint can be described as

$$
f(i, j) \geq H \quad i \in[1, N(j)], j \in[1, M] .
$$

There are some factors which should be considered as follows.

For the mobile users, the remaining energy should be considered at first. Only when one mobile user's remaining energy is higher than the threshold could the mobile user carry out the task of spectrum sensing. Let Th be the normalized threshold of the remaining energy, $\mathbf{K}$ denote the set of all mobile users, and $e_{k}$ be the remaining energy for a mobile user $k$. Then the energy constraint can be expressed as

$$
e_{k} \geq \text { Th } k \in \mathbf{K} .
$$

Let $M$ denote the number of locations needed to be sensed. For a location $j$, only the mobile users in that location can sense the channels within that location. We assume a mobile user can only sense one channel. In location $j$, let $\mathbf{K}(j)$ denote the set of mobile users, $n(j)$ denote the number of mobile users, and $N(j)$ denote the number of channels that should be sensed. For the mobile user $k \in \mathbf{K}(j), x_{k i}=1$ denotes that the channel $i$ is sensed by mobile user $k$ and $x_{k i}=0$ denotes that the channel $i$ is not sensed by mobile user $k$. Then considering a mobile user can only sense one channel, another constraint can be expressed as

$$
\sum_{k \in \mathbf{K}(j)} \sum_{i=1}^{N(j)} x_{k i} \leq n(j) .
$$

Additionally, the incentive scheme allows FC to pay for the mobile users that try to sense channels. However, the cost of crowdsensing must be in the acceptable range. Let $C$ be the maximum cost that can be paid for the sensing users and $c_{k}$ be the cost for the mobile user $k \in \mathbf{K}(j)$. The constraint can be expressed as

$$
\sum_{j=1}^{M} \sum_{k \in \mathbf{K}(j)} c_{k} \sum_{i=1}^{N(j)} x_{k i} \leq C .
$$

The optimal object of crowdsensing task assignment can be described as

$$
\max \sum_{j=1}^{M} \sum_{i=1}^{N(j)} w_{j}^{i} f(i, j)
$$

$$
\begin{array}{ll}
\text { subject to } & f(i, j) \geq H, \quad i \in[1, N(j)], j \in[1, M] \\
& e_{k} \geq \mathrm{Th}, \quad k \in \mathbf{K} \\
& \sum_{k \in \mathbf{K}(j)} \sum_{i=1}^{N(j)} x_{k i} \leq n(j) \\
& \sum_{j=1}^{M} \sum_{k \in \mathbf{K}(j)} c_{k} \sum_{i=1}^{N(j)} x_{k i} \leq C \\
& \sum_{j=1}^{M} \sum_{i=1}^{N(j)} w^{i}{ }_{j}=1 .
\end{array}
$$

Figure 1 depicts an example of crowdsensing task assignment. There are two locations and three channels in the system. Each location is divided into three sublocations. Mobile users in different sublocations may obtain different sensing results about the same channel. Since the local constraint is not satisfied or the remaining energy is not enough or the cost is too high, some mobile users are not assigned sensing task. Other users are assigned channels to sense according to formula (6).

3.2. NP-Hardness. The problem of crowdsensing task assignment is difficult to solve since we prove this problem is NPhard. The reason is that the problem of crowdsensing task assignment is as hard as maximum coverage problem which is NP-hard [21].

The maximum coverage problem is described as follows: given a number $d$ and a collection of $l$ sets $S=\left\{S_{1}, S_{2}, \ldots, S_{l}\right\}$, 


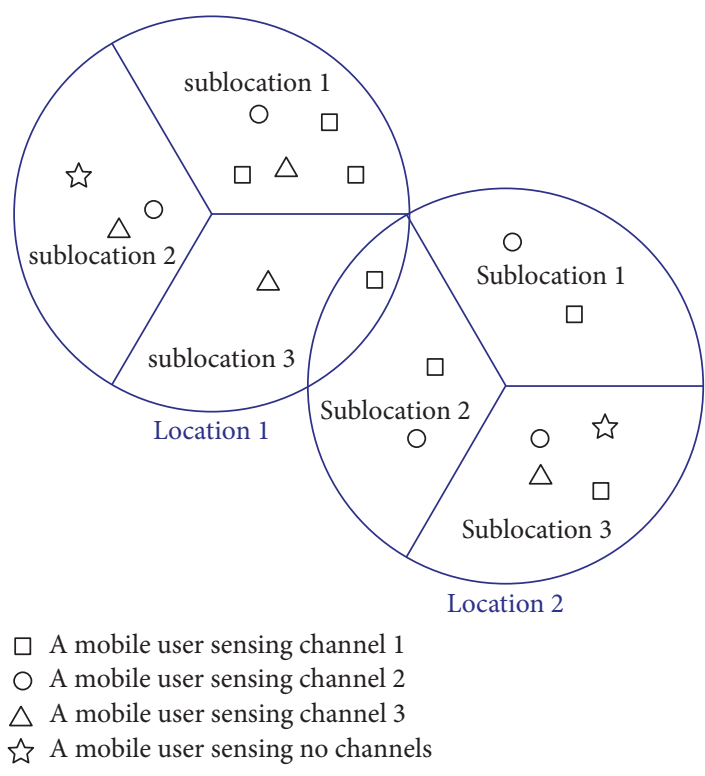

FIGURE 1: An example of crowdsensing task assignment.

the maximum coverage problem is to select at most $d$ of these sets to form $S^{\prime}$ such that the maximum number of elements is covered:

$$
\begin{array}{ll}
\max _{S^{\prime}} & \left|\bigcup_{S_{i} \in S^{\prime}} S_{i}\right|, \\
\text { s.t. } & S^{\prime} \subseteq S, \quad\left|S^{\prime}\right| \leq d .
\end{array}
$$

Theorem 1. The problem of crowdsensing task assignment is NP-hard.

Proof. By showing a special case of crowdsensing task assignment is as hard as maximum coverage problem, we prove that the problem of crowdsensing task assignment is NP-hard.

The special case is described as follows: each mobile user has enough energy to carry out spectrum sensing, the local threshold $H$ is set to zero that means the local constraint is satisfied, and the cost of crowdsensing is in the acceptable range. There are $K$ mobile users and $M$ locations in the system. And, in each location, there are $N$ channels that should be sensed. Each mobile user is denoted by $i \in$ $\{1,2, \ldots, K\}$. Then $K$ mobile users can form $2^{K}$ sets such as $\{1\},\{1,2\}$, and $\{1,2,3\}$. Let the nonnegative weight $w_{j}^{i}$ be a constant. Then (6) can be rewritten as

$$
\max \sum_{j=1}^{M} \sum_{i=1}^{N} f(i, j) .
$$

Let $l$ equal $2^{K}$ and $d$ equal MN. Equation (8) means selecting $d$ sets from $l$ sets to maximize the sum of $f(i, j)$. Compared to (7), it is at least as hard as the maximum coverage problem which is NP-hard. In other words, the special case of crowdsensing task assignment is NP-hard.

The problem of crowdsensing task assignment is no easier than the special case. Therefore, the problem of crowdsensing task assignment is NP-hard.

\section{The Optimal Algorithm Based on PSO}

Since the crowdsensing task assignment problem is NP-hard, we design the optimal algorithm based on particle swarm optimization (PSO) to solve this problem in this section. The PSO algorithm is good at NP-hard problem optimization [22]. The PSO algorithm is described at first. Then the optimal algorithm based on PSO is proposed. And time complexity is analyzed.

4.1. PSO Algorithm. In the PSO algorithm [23], each particle flies in the search space with certain speed. During the flight, a particle changes its flight experience with its companions. Therefore, each particle can fly to a better solution region based on this mechanism. Let $V_{i d}$ denote the particle speed and $X_{i d}$ denote the particle's position. The movement of the particle is described as follows:

$$
\begin{aligned}
& V_{i d}^{t+1}=w V_{i d}^{t}+c_{1} r_{1}\left(P_{i d}-X_{i d}^{t}\right)+c_{2} r_{2}\left(P_{g d}-X_{i d}^{t}\right) \\
& X_{i d}^{t+1}=V_{i d}^{t+1}+X_{i d}^{t},
\end{aligned}
$$

where $w$ denotes the inertia weight, $P_{i d}$ denotes this particle's historical best position, and $P_{g d}$ denotes the global best position. Both $r_{1}$ and $r_{2}$ are independent in the range $[0,1]$, and both $c_{1}$ and $c_{2}$ are study factors. The inertia weight $w$ makes the algorithm improve its performance according to a series of applications. Formulas (9) and (10) calculate the current particle's velocity and position, respectively.

4.2. Crowdsensing Task Assignment Algorithm Based on PSO. We design an optimal algorithm based on PSO to solve crowdsensing task assignment. According to PSO algorithm, each particle's position represents a solution to the crowdsensing task assignment problem. It can be denoted by a matrix as follows.

When there are $N(j)$ channels in location $j \in[1, M]$, the total number of sensing channels is $\sum_{j=1}^{M} N(j)$ in all locations. Let $K$ denote the number of mobile users. Then each particle is defined as a $K \times \sum_{j=1}^{M} N(j)$ matrix $\mathbf{X}$, where $\mathbf{X}[a][b]=1$ denotes that the mobile user $a$ chooses channel $b$ to sense, and $\mathbf{X}[a][b]=0$ denotes that the mobile user $a$ does not choose channel $b$ to sense.

We optimize the crowdsensing task assignment based on PSO algorithm (PSO-CTA). The optimized algorithm is described as follows. Initialize $q$ particles randomly, and each particle denotes a solution of crowdsensing task assignment of all $K$ mobile users. Then we set the particle with the highest objective function based on formulas (6) to be the current best solution. According to the PSO algorithm, we use the PSO formulas (9) to merge the crowdsensing task assignment and determine the new particle position until it converges or this swarm obtains its longest lifetime. If PSO-CTA converges, the best solution can be obtained. The proposed algorithm is described as follows. 
Initialization. The first important problem to be solved is how the algorithm initially produces the particles. We produce a random particle as follows.

For a mobile user, its remaining energy should be consid ered at first. If its remaining energy is higher than the threshold, the mobile user could carry out the task of spectrum sensing. Then it chooses a channel to sense randomly in its corresponding locations. All mobile users with enough energy choose channels like this. If the local constraint of sensing effect in (2) is satisfied in each location, the cost should be considered next. Otherwise, this particle should be generated again. If the cost for the mobile users which carry out spectrum sensing is lower than the maximum cost $C$, the process of initialization is completed. If the cost for the mobile users which carry out spectrum sensing is higher than the maximum cost $C$, FC will not assign sensing task to some mobile users to satisfy the cost constraint. At first, when there are multiple users sensing a channel in the same sublocation, FC will only choose a user with lower cost to assign sensing task, and other users are given up. According to our model, the sensing effect will not change. If the cost constraint is satisfied, the initialization is completed. Otherwise, FC should continue to give up users in the sublocations with less weight until the cost constraint is satisfied. Then a $K \times \sum_{j=1}^{M} N(j)$ matrix $\mathbf{X}$ is generated corresponding to this particle.

Initialize $q$ particles randomly, and each particle denotes a solution of crowdsensing task assignment of all $K$ mobile users.

Optimizing Process. After each spectrum sensing instance of a mobile user, its energy will decrease. A mobile user should determine that its remaining energy meets the energy constraint. If its remaining energy is higher than the threshold, the mobile user is able to carry out spectrum sensing again. If its remaining energy is lower than the threshold, the mobile user could not carry out spectrum sensing from now on. For each particle, if a mobile user's energy is not enough to carry out spectrum sensing, the user's row vector is set to zero in the corresponding matrix. Then the matrix of a particle will change.

Based on the current matrix, the crowdsensing effect function of the particle is obtained following (1). After calculating all particles' effect function, we can derive a particle's historical best position $P_{i d}$ and the global best position $P_{g d}$. The best position corresponds to the maximized crowdsensing effect function.

According to a particle's historical best position $P_{i d}$ and the global best position $P_{g d}$, we merge the matrixes to optimize the sensing task assignment. Let $\mathbf{T}_{1}$ denote the current matrix of a particle and $\mathbf{T}_{2}$ and $\mathbf{T}_{3}$ denote historical best solution of the particle and the global best solution, respectively. The merging matrix can be described as the combination of $\mathbf{T}_{1}, \mathbf{T}_{2}$, and $\mathbf{T}_{3}$. Then we optimized the merging matrix as follows.

In the merging matrix, if a channel in a sublocation is sensed by multiple users, only one user with higher energy is reserved and other users are given up. That means only an element is set to one in the column vector of the merging matrix after optimization. If a user chooses different channels to sense in $\mathbf{T}_{1}, \mathbf{T}_{2}$, and $\mathbf{T}_{3}$, there are more than elements set to one in the row vector of the merging matrix. Considering the global property of PSO, we optimize the row vectors of the merging matrix with specific probability decided by the parameters in (9) to guarantee the search space. If a mobile user chooses different channels in these three matrixes, the user will select the channel in $\mathbf{T}_{\mathbf{1}}$ based on the probability $w /\left(w+c_{1}+c_{2}\right)$, select the channel in $\mathbf{T}_{2}$ based on the probability $c_{1} /\left(w+c_{1}+c_{2}\right)$, and select the channel in $\mathbf{T}_{3}$ based on the probability $c_{2} /\left(w+c_{1}+c_{2}\right)$. That means only an element is set to one in the row vector of the merging matrix after optimization. The search space and converging speed of this algorithm can be adjusted by adjusting the values of $w, c_{1}$, and $c_{2}$.

The proposed algorithm for crowdsensing task assignment problem is described in Algorithms 1, 2, and 3.

4.3. Analysis of Time Complexity. The complexity of proposed PSO-CTA algorithm is computed as follows. The computation complexity is $O(n \times N \times q)$ in the initialization stage, where $n$ denotes the number of mobile users, $N$ denotes the number of channels, and $q$ denotes the number of particles.

In Line (3) of Algorithm 1, optimizing the sensing task assignment which is described in Algorithm 3 dominates the complexity of our algorithm. Then we focus on the computation complexity of optimizing the sensing task assignment. In a particle, the mobile users satisfying formulas (2), (3), (4), and (5) should be chosen, and the chosen mobile users are combined to obtain the maximized sensing effect function. Therefore, the complexity of a particle is $O\left(n^{2} \times N\right)$. The complexity of all particles is $O\left(n^{2} \times N \times q\right)$ at the stage of evaluating sensing effect function.

When particles update their velocities and positions, the computation complexity is $O(n \times q)$ in Lines (4)-(7) of Algorithm 1. Therefore, the computation complexity of the whole algorithm is $O\left(n^{2} \times N \times q\right)$.

\section{Simulation Results}

The proposed PSO-CTA algorithm is evaluated by simulations. The average solution is obtained by running the algorithm 100 times. We compare our PSO-CTA algorithm with the algorithm in [14]. The simulation parameters are described as follows. There are some locations needed to be sensed, with the same radius. Each location is equally divided into 3 sublocations. The whole number of channels is $N=5$. The local threshold $H$ is set to 0.57 . The nonnegative weight of $w^{i}{ }_{j}$ is identical for each channel and each location. Mobile users are deployed randomly in the locations.

Figure 2 shows the crowdsensing effect outcomes as the number of locations varies from 15 to 40 when there are 50 mobile users. The cost values of $c_{k}$ are chosen from $\{1 ; 2 ; 3 ; \ldots ; 49 ; 50\}$. The maximum cost is $C=a \sum_{k=1}^{50} c_{k}$, where $a$ equals 0.6 and 0.8 , respectively. The normalized energy threshold of Th is set to 0.2 and 0.5 , respectively. The crowdsensing effect function could be obtained based 


\section{Input:}

Objective function according to formula (6);

A local constraint $H$;

The number of mobile users $K$;

The number of locations $M$;

The number of channels $N(j)$ in location $j$;

The number of sub-locations $m(j)$ in location $j$;

The maximum cost $C$;

The maximal generation $T$;

Output: The maximum sensing effect function and sensing task assignment

\section{Initialization:}

Randomly generate each particle;

Optimization:
(1) repeat
(2) for each particle
(3) Optimizing the crowdsensing task assignment of the particle;
(4) Update the $P_{i d}$;
(5) Update the $P_{g d}$;
(6) end for
(7) until stopping criterion is satisfied

Algorithm 1: Overall procedure of proposed PSO-CTA.
(1) for each mobile user
(2) if its remaining energy satisfies formula (3)
(3) It chooses a random channel;
(4) else it will not sense;
(5) end if
(6) end for
(7) if the cost constraint is satisfied
(8) The initialization is completed;
(9) else reserve a user sensing a same channel in a sub-location;
(10) end if
(11) if the cost constraint is satisfied
(12) The initialization is completed;
(13) else give up users less weight until the cost is satisfied;
(14) end if

Algorithm 2: Random generation of each particle (initialization).

(1) for each particle

(2) for each mobile user

(3) if its remaining energy satisfies formula (3)

(4) Maintain the matrix;

(5) else set the corresponding row vector to zero;

(6) end if

(7) end for

(8) Then the current matrix $\mathbf{T}_{1}$ is derived;

(9) Evaluate the crowdsensing effect function of this particle;

(10) Obtainthis particle's $P_{i d}$ (matrix $\mathbf{T}_{2}$ ) based on crowdsensing effect function;

(11) Obtain $P_{g d}$ (matrix $\mathbf{T}_{3}$ ) with the optimal $P_{i d}$;

(12) Merge matrix $\mathbf{T}_{1}, \mathbf{T}_{2}, \mathbf{T}_{3}$;

(13) Optimize the column vectors of the merging matrix;

(14) Optimize row vectors of the merging matrix with specific probability using (9);

(15) Evaluate the crowdsensing effect function of merging matrix according to (6);

(16) end for

Algorithm 3: Procedure of optimizing the sensing task assignment of the particle. 


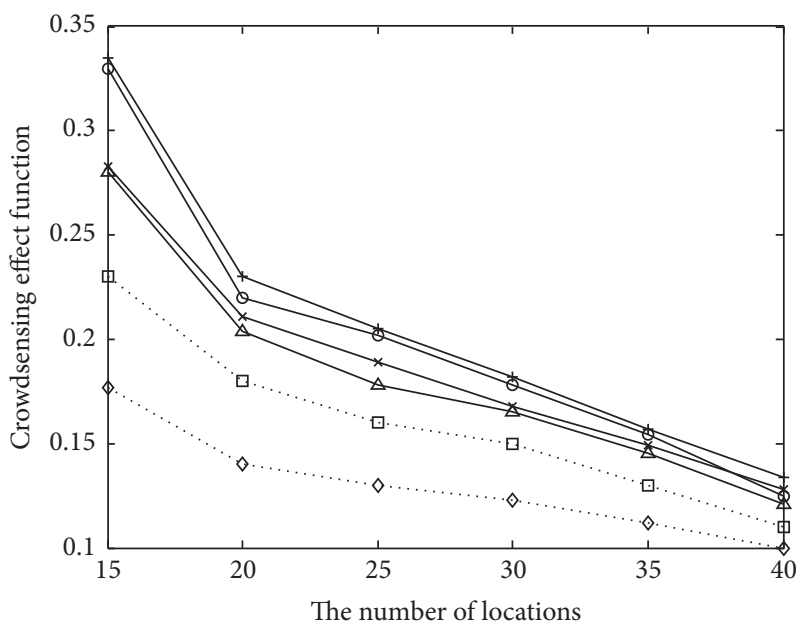

$$
\begin{aligned}
& \text { - }- \text { PSO-CTA, Th }=0.2, a=0.6 \\
& \text { — PSO-CTA, Th }=0.2, a=0.8 \\
& \triangle \text { PSO-CTA, Th }=0.5, a=0.6 \\
& \rightarrow \text { PSO-CTA, Th }=0.5, a=0.8 \\
& \text { ๑. The budget algorithm, } a=0.8 \\
& \diamond \diamond . \text { DRA, } a=0.8
\end{aligned}
$$

FIGURE 2: Crowdsensing effect function with 50 mobile users.

on (1). This function reflects the sensing effect and its value is between 0 and 1 . The closer the value is to 1 , the better the sensing effect is. Compared to the DRA algorithm in [13] and the budget algorithm in [14], our proposed PSO-CTA algorithm achieves higher crowdsensing effect function. As the number of locations increases, the crowdsensing effect function decreases. The reason is that more locations lead to more sublocations and fixed number of mobile users cannot sense all sublocations. When Th equals 0.2 and $a$ equals 0.8 , the crowdsensing effect function obtained is higher than those obtained when Th and $a$ equal other values, since there are more mobile users assigned to sense channels with $\mathrm{Th}=$ 0.2 and $a=0.8$.

Figure 3 shows the crowdsensing effect results as the number of mobile users varies from 20 to 70 when there are 20 locations. The cost values of $c_{k}$ are chosen from $\{1 ; 2 ; 3 ; \ldots ; L\}$, where $L$ denotes the number of mobile users. The maximum cost is $C=a \sum_{k=1}^{L} c_{k}$, where $a$ equals 0.6 and 0.8 , respectively. The normalized energy threshold of Th is set to 0.2 and 0.5 , respectively. Compared to the DRA algorithm in [13] and the budget algorithm in [14], our proposed PSO-CTA algorithm achieves higher crowdsensing effect function. As the number of mobile users increases, the crowdsensing effect function increases. The reason is that more sublocations could be sensed by more mobile users. When Th equals 0.2 and $a$ equals 0.8 , the crowdsensing effect function obtained is higher than those obtained when Th and $a$ equal other values, since there are more mobile users assigned to sense channels with $\mathrm{Th}=0.2$ and $a=0.8$.

Figure 4 shows the average remaining energy of mobile users as the number of spectrum sensing instances increases when there are 50 mobile users deployed randomly in 15 locations. It is assumed that the initial average energy of

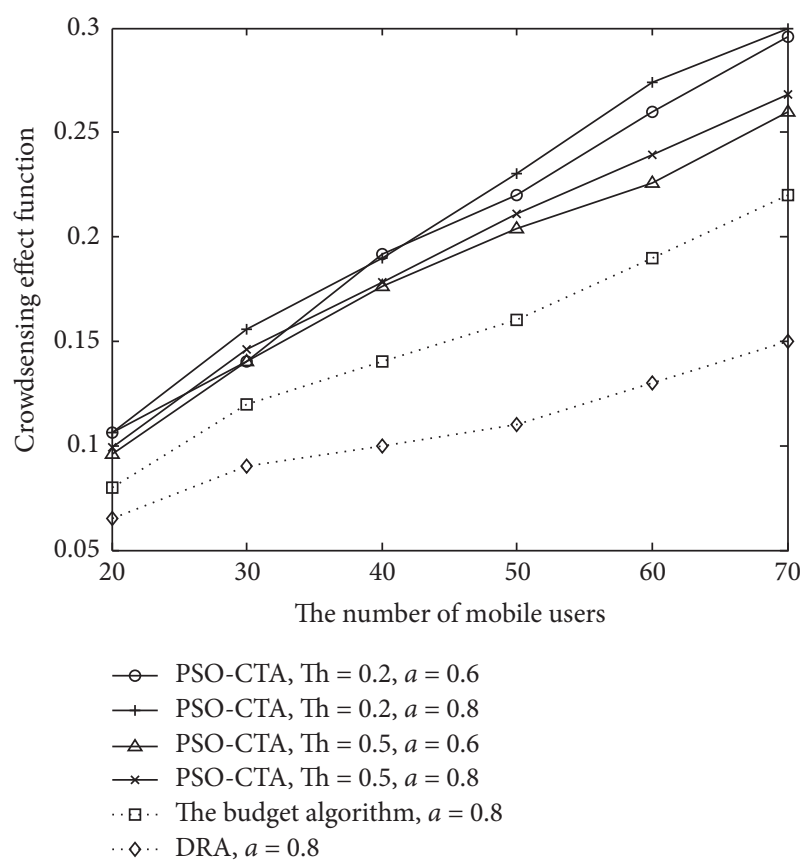

FIGURE 3: Crowdsensing effect function with 20 locations.

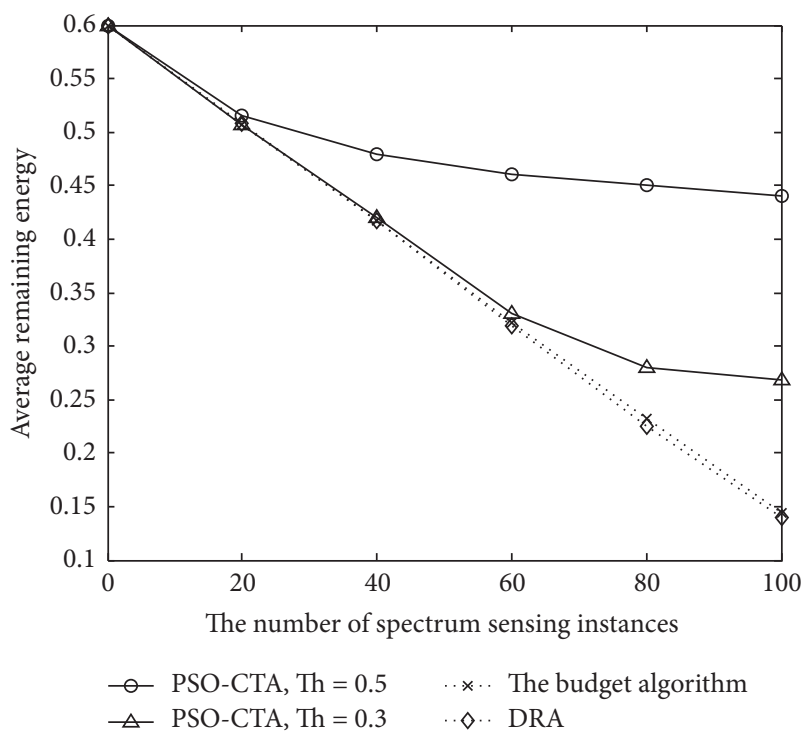

FIGURE 4: Average remaining energy with the number of spectrum sensing instances.

each user is 0.6. And after each spectrum sensing instance, a mobile user's energy falls $0.5 \%$. The normalized energy threshold Th is set to 0.5 and 0.3 , respectively. As shown in Figure 4, our proposed PSO-CTA algorithm achieves higher remaining energy of mobile users than the other algorithms. And we can see that the remaining energy will be higher when the threshold of Th is set to a higher value.

It is assumed that there are four channels and three locations which can be divided into three sublocations. The nonnegative weight is not identical for each channel. We 


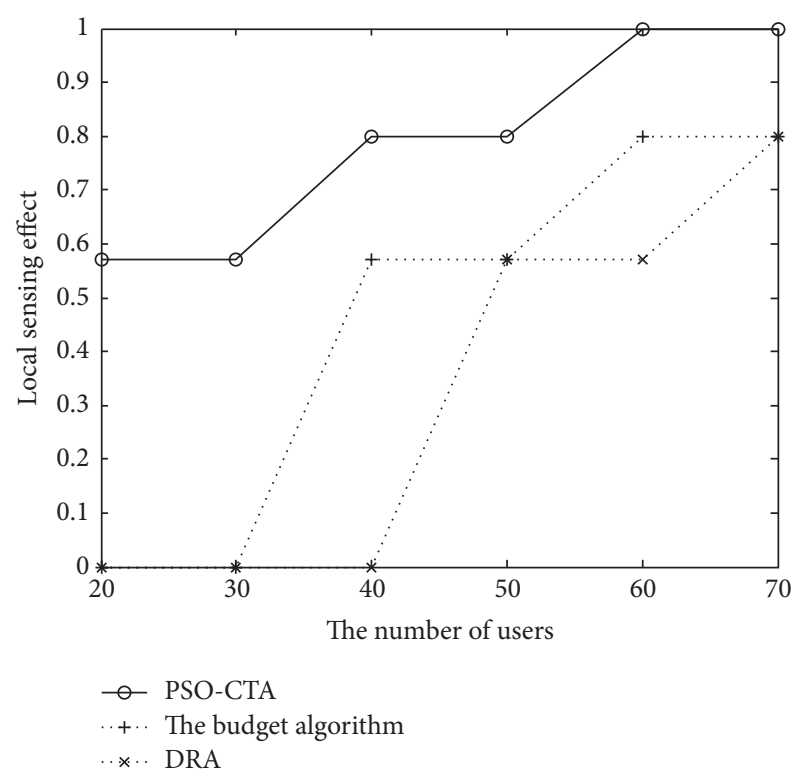

FIGURE 5: Local sensing effect with weight equaling 0.1 for three locations.

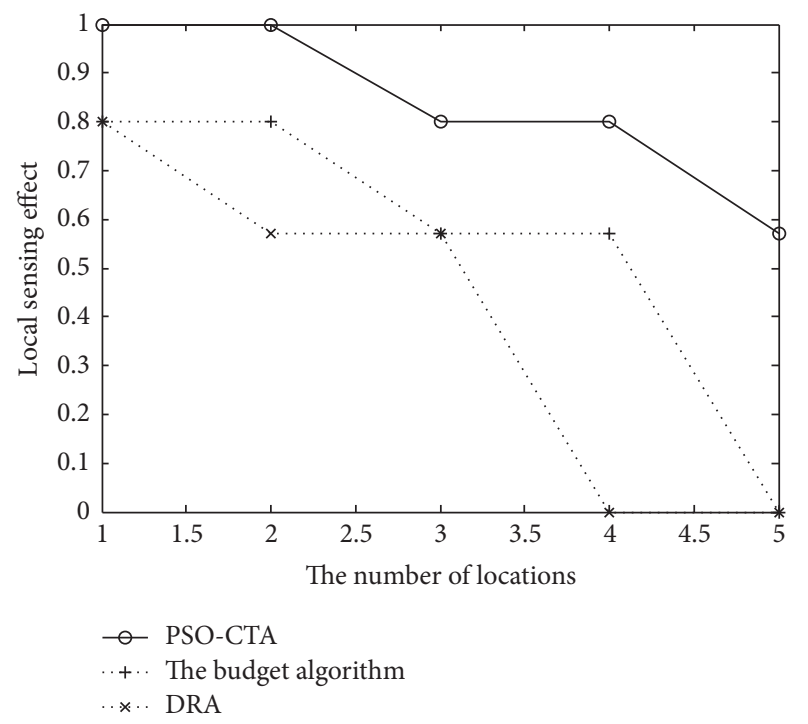

FIGURE 6: Local sensing effect with weight equaling 0.1 for 40 users.

set the weights equal to $0.3,0.3,0.3$, and 0.1 for these four channels. Under the aforementioned conditions, the local sensing effect for the fourth channel (weight equaling 0.1) is shown in Figure 5. When there are not so many users in the system, the fourth channel is not sensed for the budget algorithm and DRA algorithm as the weight is too small to increase the global sensing effect. In the proposed PSOCTA algorithm, the fourth channel should be sensed because a local constraint is set. Any channel, no matter what its weight equals, should be sensed. Therefore, no channel will be omitted with the PSO-CTA algorithm.

When there are 40 users, Figure 6 shows the local sensing effect for the fourth channel as the number of locations increases. There will not be enough users to sense each channel if the number of locations increases. Thus, the budget algorithm and DRA algorithm may choose the channels with higher weights to improve the global sensing effect. Therefore, the local sensing effect for the fourth channel will decrease. However, the proposed PSO-CTA algorithm will not ignore the fourth channel due to the local constraint.

\section{Conclusion}

For a large-scale region, this paper proposes the crowdsourcing method to assign the spectrum sensing task to many mobile users such as smartphones and pads. Considering some constraints such as remaining energy, locations, and costs of mobile users, we propose a sensing effect function with a local constraint and aim to maximize the sensing effect function. Since the problem of sensing task assignment is proved to be NP-hard, we design an optimal algorithm based on PSO to solve this problem. Simulation results show our algorithm achieves higher performance than the other algorithms.

\section{Conflicts of Interest}

The authors declare that there are no conflicts of interest regarding the publication of this paper.

\section{Acknowledgments}

This study is supported in part by National Natural Science Foundation of China (no. 61402270), Natural Science Foundation of Shandong Province, China (nos. BS2015DX003, ZR2014FQ009), Key Research and Development Program of Shandong Province, China (no. 2017GGX10142), and China Postdoctoral Science Foundation (no. 2014M561930).

\section{References}

[1] J. Mitola III and G. Q. Maguire Jr., "Cognitive radio: making software radios more personal," IEEE Personal Communications, vol. 6, no. 4, pp. 13-18, 1999.

[2] B. Guo, Z. Wang, Z. Yu et al., "Mobile crowd sensing and computing: the review of an emerging human-powered sensing paradigm," ACM Computing Surveys, vol. 48, no. 1, article 7, 2015.

[3] Y. Saleem and M. H. Rehmani, "Primary radio user activity models for cognitive radio networks: a survey," Journal of Network and Computer Applications, vol. 43, pp. 1-16, 2014.

[4] T. Yücek and H. Arslan, "A survey of spectrum sensing algorithms for cognitive radio applications," IEEE Communications Surveys \& Tutorials, vol. 11, no. 1, pp. 116-130, 2009.

[5] F. Zeng, Z. Tian, and C. Li, "Distributed compressive wideband spectrum sensing in cooperative multi-hop cognitive networks," in Proceedings of the 2010 IEEE International Conference on Communications, ICC 2010, zaf, May 2010.

[6] J. Feng, G. Lu, H. Wang, and X. Wang, "Supporting secure spectrum sensing data transmission against SSDH attack in cognitive radio ad hoc networks," Journal of Network and Computer Applications, vol. 72, pp. 140-149, 2016. 
[7] R. Zhang, J. Zhang, Y. Zhang, and C. Zhang, "Secure crowdsourcing-based cooperative pectrum sensing," in Proceedings of the 32nd IEEE Conference on Computer Communications, IEEE INFOCOM 2013, pp. 2526-2534, April 2013.

[8] Y. Lu, D. Wang, and M. Fattouche, "Cooperative spectrumsensing algorithm in cognitive radio by simultaneous sensing and BER measurements," Eurasip Journal on Wireless Communications and Networking, vol. 2016, no. 1, article no. 136, 2016.

[9] C.-H. Liu, A. Azarfar, J.-F. Frigon, B. Sansò, and D. Cabric, "Robust cooperative spectrum sensing scheduling optimization in multi-channel dynamic spectrum access networks," IEEE Transactions on Mobile Computing, vol. 15, no. 8, pp. 2094-2108, 2016.

[10] J. Zhu, D. Jiang, S. Ba, and Y. Zhang, "A game-theoretic power control mechanism based on hidden Markov model in cognitive wireless sensor network with imperfect information," Neurocomputing, vol. 220, pp. 76-83, 2017.

[11] P. Arora, N. Xia, and R. Zheng, "A Gibbs sampler approach for optimal distributed monitoring of multi-channel wireless networks," in Proceedings of the 54th Annual IEEE Global Telecommunications Conference: "Energizing Global Communications", GLOBECOM 2011, December 2011.

[12] D.-H. Shin, S. Bagchi, and C.-C. Wang, "Distributed online channel assignment toward optimal monitoring in multichannel wireless networks," in Proceedings of the IEEE Conference on Computer Communications, INFOCOM 2012, pp. 26262630, March 2012.

[13] D.-H. Shin and S. Bagchi, "An optimization framework for monitoring multi-channel multi-radio wireless mesh networks," Ad Hoc Networks, vol. 11, no. 3, pp. 926-943, 2013.

[14] D.-H. Shin, S. He, and J. Zhang, "Joint sensing task and subband allocation for large-scale spectrum profiling," in Proceedings of the 34th IEEE Annual Conference on Computer Communications and Networks, IEEE INFOCOM 2015, pp. 433-441, 2015.

[15] J. Wang, Y. Wang, D. Zhang, F. Wang, Y. He, and L. Ma, "Psaiiocator: Multi-task allocation for participatory sensing with sensing capability constraints," in Proceedings of the 2017 ACM Conference on Computer Supported Cooperative Work and Social Computing, CSCW 2017, pp. 1139-1151, 2017.

[16] H. Chen, L. Liu, T. Novlan, J. D. Matyjas, B. L. Ng, and J. Zhang, "Spatial Spectrum Sensing-Based Device-to-Device Cellular Networks," IEEE Transactions on Wireless Communications, vol. 15, no. 11, pp. 7299-7313, 2016.

[17] R. Sun, Y. Wang, X. Wang, and Y. Zhang, "Transceiver design for cooperative non-orthogonal multiple access systems with wireless energy transfer," IET Communications, vol. 10, no. 15, pp. 1947-1955, 2016.

[18] M. Jo, T. Maksymyuk, B. Strykhalyuk, and C.-H. Cho, "Deviceto-device-based heterogeneous radio access network architecture for mobile cloud computing," IEEE Wireless Communications, vol. 22, no. 3, pp. 50-58, 2015.

[19] X. Lin, J. G. Andrews, and A. Ghosh, "Spectrum sharing for device-to-device communication in cellular networks," IEEE Transactions on Wireless Communications, vol. 13, no. 12, pp. 6727-6740, 2014.

[20] Q. Liu, X. Wang, and Y. Cui, "Robust and adaptive scheduling of sequential periodic sensing for cognitive radios," IEEE Journal on Selected Areas in Communications, vol. 32, no. 3, pp. 503-515, 2014.

[21] D. S. Hochbaum, Approximation Algorithm for NP-Hard Problems, PWS Publishing Company, Massachusetts, 1997.
[22] J. Kennedy and R. Eberhart, "Particle swarm optimizatio," in Proceedings of the IEEE International Conference on Neural Network, pp. 1942-1948, Perth, Australia, 1995.

[23] R. C. Eberhart and J. Kennedy, "A new optimizer using particle swarm theory," in Proceedings of the 6th International Symposium on Micro Machine and Human Science (MHS '95), pp. 3943, Nagoya, Japan, 1995. 


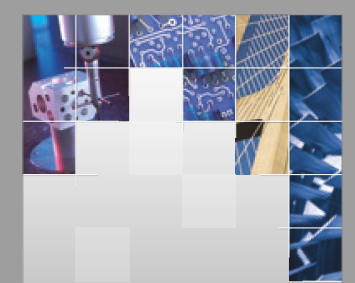

\section{Enfincering}
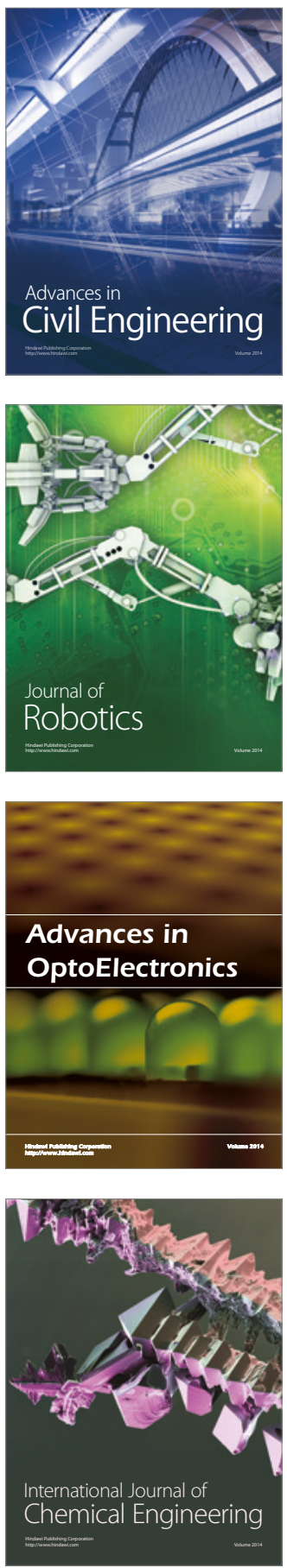

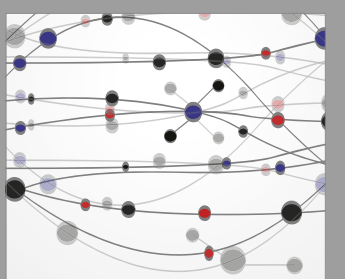

The Scientific World Journal

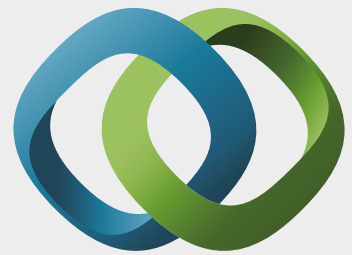

\section{Hindawi}

Submit your manuscripts at

https://www.hindawi.com
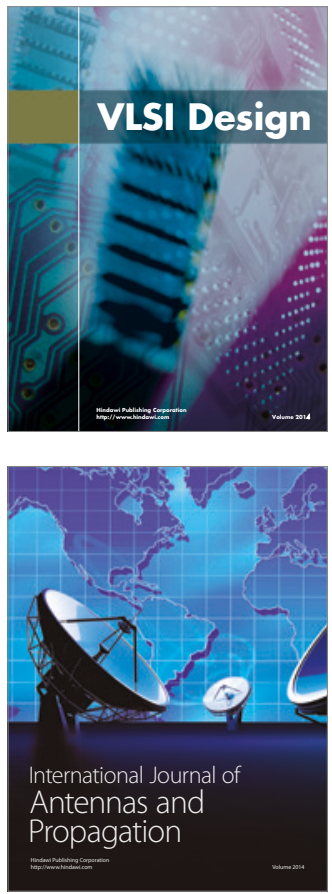

\section{Rotating}

Machinery
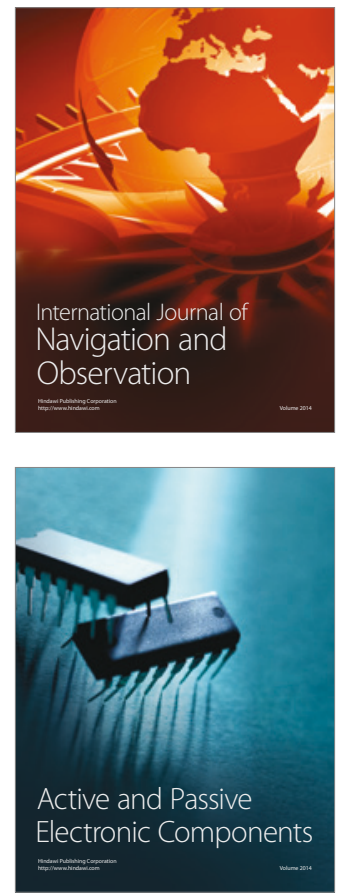
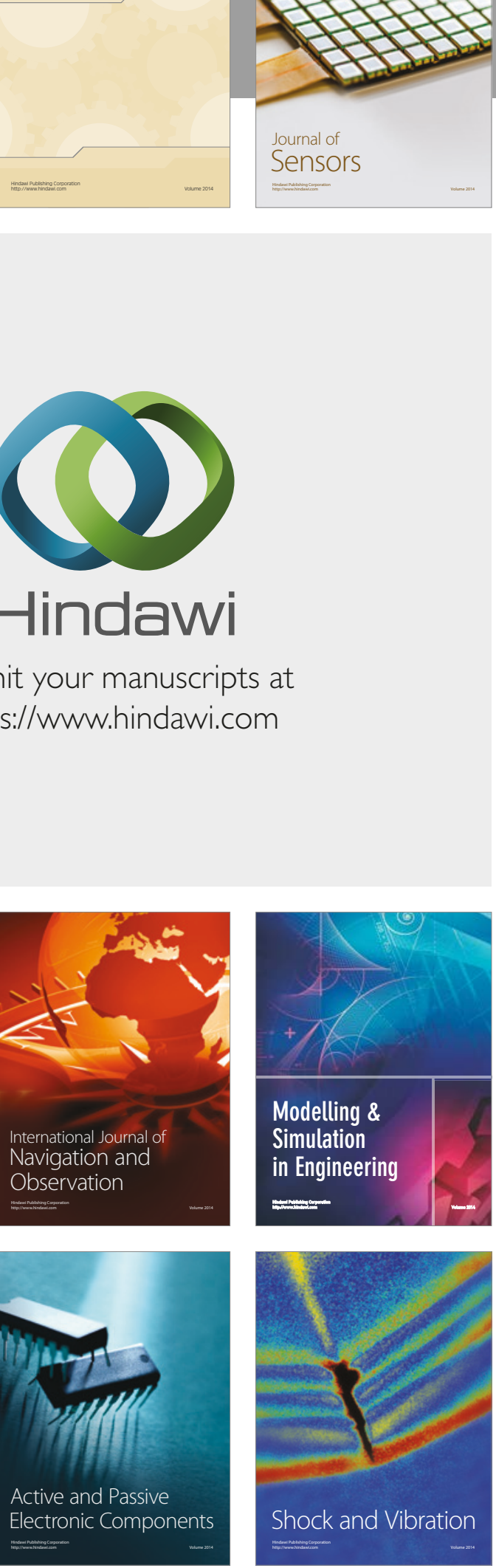
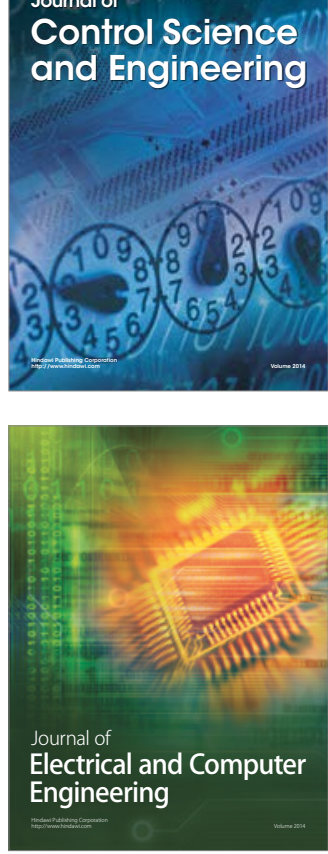

Distributed

Journal of

Control Science

and Engineering
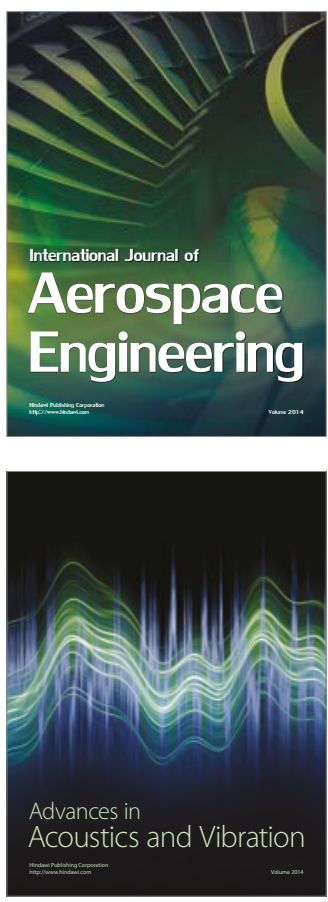

Sensor Networks 